\title{
Aneurysm Arising at the Origin of a Duplicated Middle Cerebral Artery
}

\author{
Jean Roch Alliez ${ }^{a} \quad$ Luis Manera ${ }^{b}$ \\ aDepartment of Neurosurgery, CHP Clairval, Marseille, France; bepartment of \\ Neuroradiology, CHU E. Herriot, Lyon, France
}

Keywords

Aneurysm · Duplicated middle cerebral artery · Embolization · Origin · Rupture

\section{Abstract}

A duplicated middle cerebral artery (DMCA) is a common anomaly. However, aneurysms arising from the origin of a DMCA are extremely rare. A 22-year-old female was admitted to our hospital with a World Federation of Neurosurgical Societies grade 2 subarachnoid haemorrhage. Four-vessel angiography revealed a DMCA and an aneurysm arising from the origin of this artery. The aneurysm was successfully treated by embolization, and the patient was discharged 2 weeks later. Ruptured aneurysms arising from the origin of a DMCA can be successfully treated by embolization. These aneurysms are small and 3D-computed tomography reconstruction is mandatory to detect them. It is important to preserve the DMCA during the treatment procedure.

\section{Introduction}

The 2 most common anomalies of the middle cerebral artery (MCA) are duplicated MCAs (DMCAs) and accessory MCAs (AMCAs). These anomalous arteries course along the Sylvian fissure along with the M1 segment of the MCA. DMCAs arise from the internal carotid artery (ICA) and AMCAs originate from the anterior cerebral artery. DMCAs are classified into 2 types according to their point of origin. Type A DMCAs originate at the top of the ICA and type $B$ DMCAs arise from the ICA between the anterior choroidal artery and the ICA bifurcation. We present the second case of a ruptured aneurysm arising from the origin of a type A DMCA that was successfully treated by embolization. 

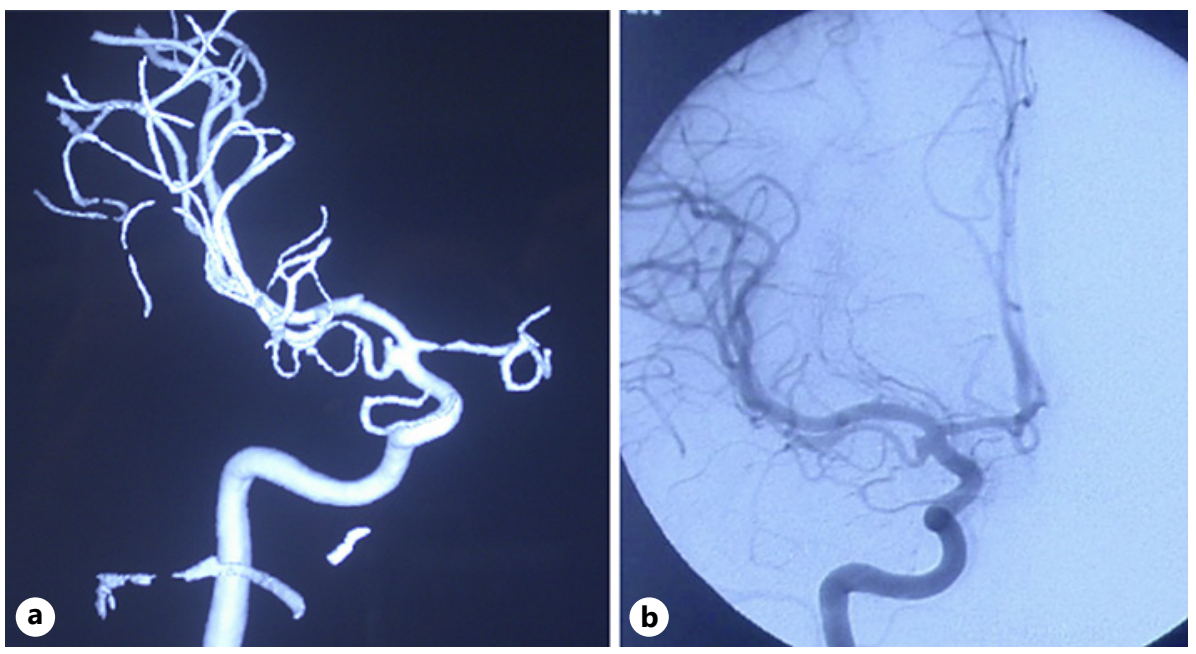

Fig. 1. Right three quarter (a) and anterior (b) carotid angiography showing the DMCA and aneurysm arising at its origin. DMCA, duplicated middle cerebral artery.

Fig. 2. Final right carotid angiography. Anterior view.

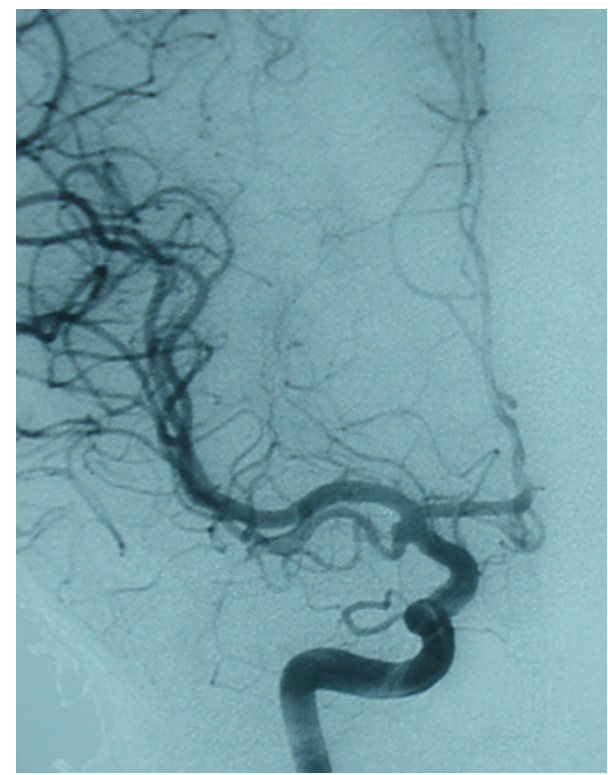

\section{Case Report}

Our patient was a 22-year-old female who was admitted to hospital with a severe acute headache, nausea, and vomiting. Neurological examination revealed nuchal stiffness and no deficit (World Federation of Neurosurgical Societies grade 2). A computed tomography (CT) scan of the brain was performed immediately, disclosing a high-density area in the basal cisterns, right Sylvian fissure and fourth ventricle, and mild dilation of the ventricular system. Traditional digital subtraction angiography revealed a duplication of the MCA and a 2-mm aneurysm arising from the origin of this artery (shown in Fig. 1). After emergency discussion between a neuroradiologist, neurosurgeon, and the patient, endovascular treatment was scheduled. The aneurysm was successfully excluded with a Guglielmi detachable coil (shown in Fig. 2). The patient was discharged 2 weeks later with no deficit. 


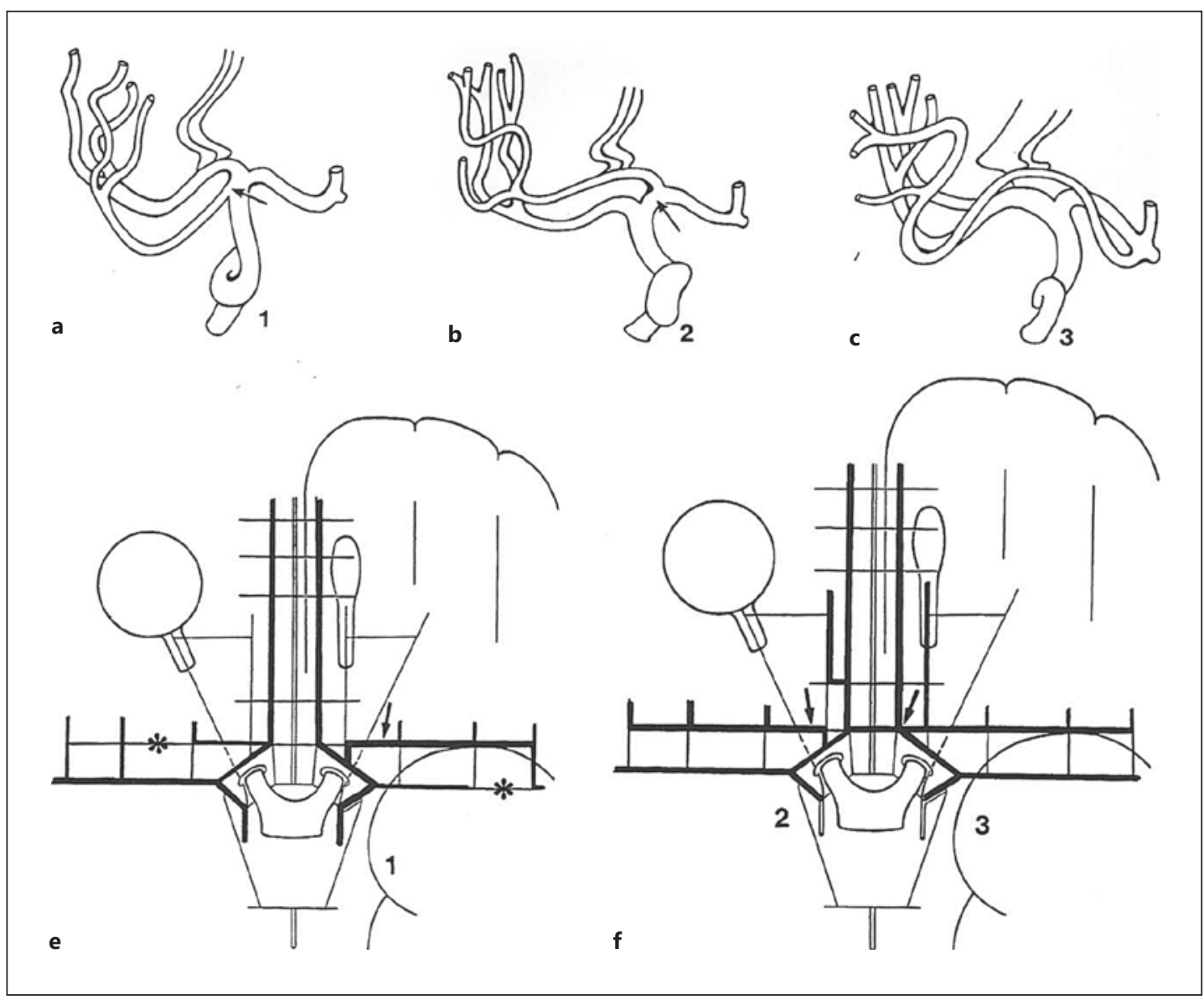

Fig. 3. Diagram of Manelfe showing different origins of an AMCA and DMCA. a Type 1: origin from the end of the carotid artery; Type 2: origin from the A1 segment (b); Type 3: origin from the end of the A1 segment, anterior communicating artery or A2 segment (c); asterisks show the artery that is involved during the embryological period and arrows show the AMCA or DMCA (d, e). DMCA, duplicated middle cerebral artery.

Written informed consent was obtained from the patient for publication of this case report and any accompanying images. The manuscript is exempt from Ethical Committee approval as it is a clinical case report and did not involve any change to the usual management of the patient.

\section{Discussion}

In 1962, Crompton reported a prevalence of anomalous arteries (AMCAs or DMCAs) of 3\%, based on 347 autopsies [1, 2]. In 1992, Yamamoto et al. [3] reviewed 455 bilateral carotid angiographies and found 14 (3\%) AMCAs and 7 DMCAs. In other autopsy-based investigations, the prevalence of DMCAs was 0.7-2.9\% and the prevalence of AMCAs was 0.3-2.7\% [2-5].

In 1973, Teal et al. [6] restricted the term AMCA to an arterial branch arising from the anterior cerebral artery (A1 or A2 segment) and coursing in the Sylvian fissure adjacent to the MCA. When the anomalous MCA, regardless of its size, arises from the ICA and runs in the Sylvian fissure, Teal et al. [6] and McCormick et al. [7] considered the term DMCA to be more appropriate. The diagram of Manelfe [8] shows the anatomical difference between the origin of a DMCA and an AMCA (shown in Fig. 3). 
Saccular aneurysms arising from the origin of a DMCA are extremely rare. The first case was reported by Crompton in 1962 in an autopsy study [1]. The patient had a ruptured ICA-DMCA aneurysm without any other vascular anomalies or cerebral aneurysms. Intracranial aneurysms in patients with a normal angio-architecture often arise from bifurcation of vessels. This seems applicable to MCA anomalies. Most aneurysms associated with an AMCA or DMCA were reported at the origins of the anomalous vessel. Notably, abnormal vasculature morphologies and haemodynamic factors of the anomalous MCA may contribute to these aneurysms. The only case featuring a saccular aneurysm arising from the DMCA trunk and not from its origin was reported by Uchino et al. [9].

To the best of our knowledge, 33 cases of aneurysm originating from a DMCA have been reported to date [10-15]. Aneurysms in this location are rare and frequently present as a subarachnoid haemorrhage despite their small size. In a recent review of 28 DMCA aneurysms, all but 1 were $<7 \mathrm{~mm}$ in size and 17 had ruptured [10]. With the exception of 2 aneurysms measuring $<2 \mathrm{~mm}$, all were treated surgically. A recent case report described a 20 -year-old female with a ruptured aneurysm at the origin of a DMCA that was successfully treated by balloon remodelling and coil embolization [15].

In the literature, 4 aneurysms originating at a DMCA were not diagnosed by conventional angiography [10]. 3D-CT reconstruction angiography is mandatory to detect these small aneurysms. In 11 cases, these DMCA aneurysms coexisted with other vascular anomalies in the brain or other aneurysms. DMCAs may play a role in supplying the blood flow to the frontal lobe and the basal ganglia through a perforating artery and this artery must not be occluded or damaged during the treatment procedure.

\section{Conclusion}

AMCAs and DMCAs are relatively well known congenital anomalies. We describe a case of a ruptured aneurysm arising at the origin of a DMCA. To our knowledge, this is only the second case of such an aneurysm to be treated successfully by embolization. These aneurysms are small, and 3D-CT reconstruction is mandatory to detect them. It is imperative to preserve the DMCA during the treatment procedure.

\section{Statement of Ethics}

Written informed consent was obtained from the patient for publication of this case report and any accompanying images. The manuscript is exempt from ethical committee approval as it is a clinical case report and did not involve any change to the usual management of the patient.

\section{Conflict of Interest Statement}

The authors have no conflicts of interest to declare.

\section{Funding Sources}

The authors did not receive any funding.

\section{Karger'}




\section{Author Contributions}

L.M. (neuroradiologist) cared for the patient and carried out the embolization. J.R.A. (neurosurgeon) wrote the manuscript.

\section{References}

1 Crompton MR, Lond MB. The pathology of ruptured middle-cerebral aneurysms with special reference to the differences between the sexes. Lancet. 1962 Sept 1;2(7253):421-5.

2 Jain KK. Some observations on the anatomy of the middle cerebral artery. Can J Surg. 1964 Apr;7:134-9.

3 Yamamoto H, Marubayashi T, Soejima T, Matsuoka S, Matsukado Y, Ushio Y. Accessory middle cerebral artery and duplication of middle cerebral artery-terminology, incidence, vascular etiology, and developmental significance. Neurol Med Chir. 1992 May;32(5):262-7.

4 Vincentelli F, Caruso G, Andriamamonji C, Rabehanta P, Graziani N, Grisoli F. Modalities of origin of the middle cerebral artery. Incidence on the arrangement of the perforating branches. J Neurosurg Sci. 1990 Jan-Mar; 34(1):7-11.

5 Umansky F, Dujovny M, Ausman JI, Diaz FG, Mirchandani HG. Anomalies and variations of the middle cerebral artery: a microanatomical study. Neurosurgery. 1988 Jun;22(6 Pt 1):1023-7.

6 Teal JS, Rumbaugh CL, Bergeron RT, Segall HD. Anomalies of the middle cerebral artery: accessory artery, duplication, and early bifurcation. Am J Roentgenol Radium Ther Nucl Med. 1973 Jul;118(3):567-75.

7 Krayenbühl H, Yasargil M. Cerebral angiography. London, UK: Butterworth; 1968. p. 59-60.

8 Lasjaunias P, Berenstein A. Surgical neuro-angiography functional vascular anatomy of brain spinal cord and spine. Springer-Verlag; 1986. Vol. 3.

9 Uchino M, Kitajima S, Sakata Y, Honda M, Shibata I. Ruptured aneurysm at a duplicated middle cerebral artery with accessory middle cerebral artery. Acta Neurochir. 2004 Dec;146(12):1373-5; discussion 1375.

10 Elsharkawy A, Ishii K, Niemelä M, Kivisaari R, Lehto H, Hernesniemi J. Management of aneurysms at the origin of duplicated middle cerebral artery: series of four patients with review of the literature. World Neurosurg. 2013 Dec;80(6):e313-8.

11 Iida Y, Tamase A, Kamide T, Mori K, Seki S, Nomura M. Aneurysm at origin of duplicated middle cerebral artery associated with another aneurysm. Surg Neurol Int. 2015 Oct;6(Suppl 21):S549-52.

12 Kim JS, Lee $\mathrm{CH}$, Park H, Han JW. An unruptured cerebral aneurysm at the origin of the duplicated middle cerebral artery. J Cerebrovasc Endovasc Neurosurg. 2015 Sept;17(3):223-6.

13 Miyoshi H, Migita K, Kumano K, Hashimoto N, Toyota A. A case of aphasia after neck clipping of a ruptured aneurysm at the origin of the duplicated middle cerebral artery. No Shinkei Geka. 2016 Nov;44(11):959-64.

14 Ren H, Ma L, Wei M, Li J, Yu M, Yin L. Duplicated middle cerebral artery origin with an aneurysm. Medicine. 2018 Mar; 97(9):e9947.

15 Tsang COA, Smith L, Klostranec J, Orru E, Pereira VM. Ruptured duplicated middle cerebral artery aneurysm successfully treated by coil embolization with balloon remodeling. World Neurosurg. 2018 Dec;120:509-10. 